\title{
Texture-Based Automatic Separation of Echoes from Distributed Moving Targets in UWB Radar Signals
}

\section{AUTHOR(S):}

Sakamoto, Takuya; Sato, Toru; Aubry, Pascal J.; Yarovoy, Alexander G.

\section{CITATION:}

Sakamoto, Takuya ... [et al]. Texture-Based Automatic Separation of Echoes from Distributed Moving Targets in UWB Radar Signals. IEEE Transactions on Geoscience and Remote Sensing 2015, 53(1): 352-361

\section{ISSUE DATE:}

2015-01

URL:

http://hdl.handle.net/2433/189731

\section{RIGHT:}

(c) 2014 IEEE. Personal use of this material is permitted. Permission from IEEE must be obtained for all other uses, in any current or future media, including reprinting/republishing this material for advertising or promotional purposes, creating new collective works, for resale or redistribution to servers or lists, or reuse of any copyrighted component of this work in other works.; この論文は出版社版でありません。引用の際には出版社版をご確認ご利用ください。; This is not the published version. Please cite only the published version. 


\title{
Texture-Based Automatic Separation of Echoes from Distributed Moving Targets in UWB Radar Signals
}

\author{
Takuya Sakamoto, Member, IEEE Toru Sato, Member, IEEE \\ Pascal J. Aubry, Member, IEEE and Alexander G. Yarovoy, Senior Member, IEEE
}

\begin{abstract}
A novel algorithm is proposed for separating multiple moving targets in radar images in the slow time-range domain. Target discrimination is based on an image texture angle that is related to the target's instantaneous velocity. The algorithm efficiency has been successfully verified for targets with variable velocities.
\end{abstract}

Index Terms-ultra wide-band radar, distributed target, texture angle, walking motion, micro Doppler

\section{INTRODUCTION}

$\mathbf{T}$ HE combination of high range resolution and Doppler information makes ultra wideband (UWB) radar an important sensor for public security and indoor surveillance systems. Micro-Doppler analysis has been shown to be a powerful tool for human detection, identification and tracking [1]-[8]. Among these, Saho et al. [9] proposed a method using micro-Doppler information to identify people based on physical characteristics. Wang and Fathy [10] measured radar echoes from a person to detect arm swings, respiration, and heartbeats. However, these conventional studies all assume that the image contains data from a single person; they need an effective algorithm for separating multiple targets in the scene.

One such technology is multiple hypothesis tracking (MHT) [11] that employs a Kalman filter and multiple hypothesis technique redesigned for human tracking. Although this technique can estimate multiple trajectories of people, each trajectory is represented as a curve that does not define the actual region corresponding to the target in the radar image. Thus, this method does not actually separate the received signals into multiple components so that single-target algorithms can be applied.

Another reason why the separation of echoes is important is related to the association of multiple echoes measured with multiple sensors. When $N$ targets are measured using $M$ receivers, there are $N !^{M-1}$ possible combinations, which can be enormous. He et al. [12] proposed an algorithm for resolving this association issue based on the mutual information between adjacent radar signals. This algorithm also requires that the echoes from multiple people in received data are separated beforehand.

In this paper, we propose a new algorithm for separating echoes from multiple persons. This method analyzes the texture of the radar image in the slow time-range domain.
Image segmentation based on texture is well-known in image processing [13]-[16]. The statistical characteristics of images are used for segmentation in these methods. This kind of approach is, however, not applicable to radar imaging in the slow time-range domain because the statistical characteristics of the echoes from different targets are almost identical. The algorithm proposed uses a texture angle that corresponds to a target's line-of-sight speed. Next, we calculate a pixelconnection map in which each pixel is connected to another pixel that has the closest texture angle. Finally, randomly distributed complex values are numerically propagated to the adjacent connected pixels. This algorithm works autonomously like a self-organizing map. It is shown to be effective even for motion-varying targets. Specifically, we demonstrate that our algorithm can successfully separate echoes from two and three people walking at different and time-varying speeds.

The rest of the paper is organized as follows. We start with a formulation of distributed targets and UWB radar signals, followed by the introduction of a new algorithm with multiple steps: calculating the image texture angle, forming a pixel connection map, and the application of the complex number propagation algorithm. Next, we demonstrate the capability of the proposed algorithm with an application to the signals from two walking people measured with a UWB radar system. Finally, we analyze the applicability of the proposed method to more complicated scenarios.

\section{Distributed Target Model and Spectrogram}

We model a distributed target as a collection of multiple scattering points moving around their centers of gravity. The micro-motion corresponds to the motion of these points relative to the center of gravity. We denote the range of the center of gravity as $\rho_{0}(t)$, where $t$ is the slowtime. The range of the $i$ th scattering point is expressed as $\rho_{0}(t)+\rho_{i}(t) \quad\left(i=1, \cdots I_{\mathrm{d}}\right)$, where $\rho_{i}(t)$ is the line-of-sight range difference between the $i$-th point and the center of gravity, and $I_{\mathrm{d}}$ is the number of scattering points in each target cluster.. Figure 1 shows the assumed model with two target clusters. Each black circle is the center of gravity of the target cluster, whereas the white circles are scattering points.

The echo amplitude for the $i$-th point is $\alpha_{i}$, and, for simplicity, propagation loss, multiple scattering, shadowing 


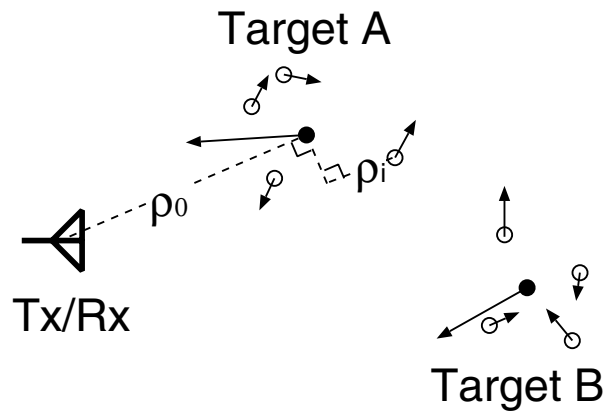

Fig. 1. The schematic of the model assumed in this section.

effect, waveform distortion caused by scattering are not considered. The received signal $s(t, r)$ for a single target cluster is expressed as

$$
s(t, r)=\sum_{i=1}^{I_{\mathrm{d}}} \alpha_{i} p\left(r-\rho_{0}(t)-\rho_{i}(t)\right),
$$

where $r$ is the range, $t$ slow time, and $p(r)$ waveform of a scattered pulse.

The Wigner-Ville distribution (WVD) is widely used to generate high-resolution spectrograms and to analyze the timedependent frequency of the dynamic signals. The WVD is known to have better time and frequency resolution than conventional methods, such as the short-time Fourier transform (STFT). The smoothed pseudo-Wigner-Ville distribution (SPWD) is a smoothed version of the WVD and has the advantage that it suppresses artifacts caused by multiple frequency components. The WVD of a signal $s(t, r)$ is expressed as

$$
W(t, \omega, r)=\int s(t+\tau / 2, r) s^{*}(t-\tau / 2, r) \mathrm{e}^{-j \omega \tau} \mathrm{d} \tau,
$$

and the SPWD is defined using the WVD as

$$
W_{\mathrm{s}}(t, \omega, r)=\iint \Phi\left(t-t^{\prime}, \omega-\omega^{\prime}\right) W\left(t^{\prime}, \omega^{\prime}, r\right) \mathrm{d} t^{\prime} \mathrm{d} \omega^{\prime},
$$

where $\Phi$ is a smoothing function, which for this paper, is a Gaussian function

$$
\Phi(t, \omega)=\exp \left(-\frac{t^{2}}{2 t_{0}^{2}}-\frac{\omega^{2}}{2 \omega_{0}^{2}}\right),
$$

with smoothing correlation lengths in terms of time $t_{0}$ and angular frequency $\omega_{0}$. To obtain a spectrogram for our study, $W_{\mathrm{s}}(t, \omega, r)$ is incoherently integrated (summed in a discrete form) regarding $r$ as

$$
W_{\text {sum }}(t, \omega)=\int\left|W_{\mathrm{s}}(t, \omega, r)\right|^{2} \mathrm{~d} r .
$$

\section{Proposed Texture Angle for Radar Echoes}

In this section, we introduce texture angle for radar images to estimate the approximate line-of-sight velocities of targets. Unlike the use of a spectrogram, the texture angle determines the Doppler velocity for each pixel of the image. In general, the echoes of different targets have different texture angles, unless those multiple targets are exactly in the same motion.
We define the texture angle of a slow time-range radar image as

$$
\theta(t, r)=\tan ^{-1}\left(v_{0} \frac{\partial s(t, r) / \partial r}{\partial s(t, r) / \partial t}\right)
$$

Note that $v_{0}$ is introduced to make the argument of $\tan ^{-1}$ dimensionless. Now we apply the texture angle to a single target case in Eq. (1) to clarify the physical meaning of the process. By partially differentiating $s(t, r)$ with regard to $r$ and $t$, one obtains

$$
\begin{aligned}
\frac{\partial s}{\partial r} & =\sum_{i=1}^{I_{\mathrm{d}}} \alpha_{i} \dot{p}\left(r-\rho_{0}(t)-\rho_{i}(t)\right) \\
\frac{\partial s}{\partial t} & =-\sum_{i=1}^{I_{\mathrm{d}}} \alpha_{i} \dot{p}\left(r-\rho_{0}(t)-\rho_{i}(t)\right)\left(\dot{\rho}_{0}(t)+\dot{\rho}_{i}(t)\right)
\end{aligned}
$$

where $\dot{p}(r)$ is the derivative of $p(r)$. If the time derivative of the micro motion $\left|\dot{\rho}_{i}(t)\right|$ is small enough, Eq. (8) take the approximate form

$$
\begin{aligned}
\frac{\partial s}{\partial t} & \simeq-\dot{\rho}_{0}(t) \sum_{i=1}^{I_{\mathrm{d}}} \alpha_{i} \dot{p}\left(r-\rho_{0}(t)-\rho_{i}(t)\right), \\
& =-\dot{\rho}_{0}(t) \frac{\partial s}{\partial r}
\end{aligned}
$$

Substituting Eqs. (7) and (9) to Eq. (6), one obtains the texture angle as

$$
\theta(t, r)=\tan ^{-1}\left(v_{0} / \dot{\rho}_{0}(t)\right),
$$

if $\frac{\partial s}{\partial t} \neq 0$. This equation shows that the texture angle is uniquely determined as a function of the line-of-sight speed of the target $\dot{\rho}_{0}(t)$. Note that the left-hand side of Eq. (11) is a function of $t$ and $r$, whereas the right-hand side is a function of only $t$. This arises from the approximation; in an actual scenario, micro-Doppler caused by $\rho_{i}(t)$ makes the right-hand side of the equation $r$-dependent. Using the texture angle, the approximate target Doppler velocity is calculated for each pixel of the image.

\section{Proposed Separation Algorithm of Echoes}

If each target has an approximately constant velocity, the echoes can be separated using a threshold (or thresholds) for the texture angle. The texture angle alone, however, cannot be applied if targets change their speed. In this section, we propose two additional methods for separating multiple echoes from targets moving at time-varying speeds.

The entire proposal consists of three steps. First, we calculate the texture angle of the signal as defined in the previous section. Second, we obtain a pixel-connection map between pixels of the texture angle image, to be explained in the next subsection. Third, we apply the connection propagation algorithm, to be described subsequently, to the pixel-connection map to separate multiple echoes. The proposed method is illustrated by a flow chart (Fig. 2). A slow time-range image is input into the procedure, while the resultant image is still in the same slow time-range domain but different targets have different values in the output image. 


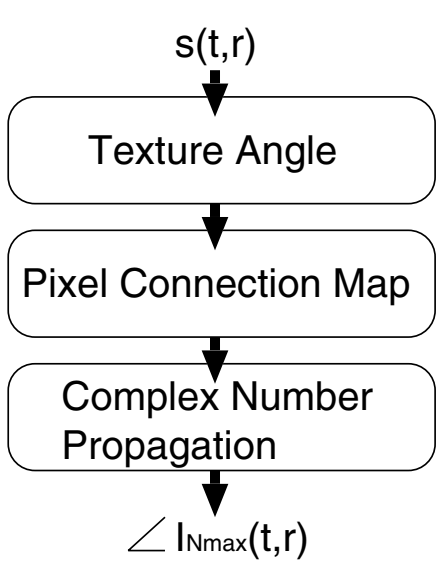

Fig. 2. Flow chart of the proposed target separation method.

\section{A. Pixel Connection Map based on Texture Angle}

Here, we explain the procedure to obtain the pixelconnection map, which corresponds to the second step of our proposed algorithm. In this pixel-connection map, each pixel is connected to another pixel that has the closest texture angle. For this calculation, we use the texture angle of each pixel as described earlier. Note that the texture angle is defined only if the intensity of the pixel is above a threshold. The following procedure applies only to pixels whose texture angle is defined. For the $i$-th pixel, the right-connected pixel is chosen as

$$
R_{i}=\arg \min _{j}\left|\theta_{j}-\theta_{i}\right|
$$

subject to

$$
t_{i}<t_{j} \leq t_{i}+T_{\mathrm{s}}
$$

and

$$
\left|\tan ^{-1}\left(\frac{v_{0}\left(t_{j}-t_{i}\right)}{r_{j}-r_{i}}\right)-\theta_{i}\right|<\delta,
$$

where $\theta_{i}, t_{i}$ and $r_{i}$ are the texture angle, slow-time and range for the $i$-th pixel.

Here, $T_{\mathrm{s}}$ is the window size for search, and $\delta$ is a small angle. These conditions imply that the pixel connected to the $i$-th pixel is located on the right hand side of the $i$-th pixel, and the inclination of the line connecting the pair of pixels is in accord with the texture angle. Under these conditions, we choose the pixel that has a texture angle closest to that of the pixel of interest.

We also calculate the second closest pixel as

$$
R_{i}^{\prime}=\arg \min _{j \neq R_{i}}\left|\theta_{j}-\theta_{i}\right|,
$$

subject to Eqs. (13) and (14).

Similarly, we calculate the left-connected pixels $L_{i}$ and $L_{i}^{\prime}$ that are located on the left-hand side of the pixel of interest using the same process Eqs. (12) and (15), but with a different time condition, $t_{i}-T_{\mathrm{s}} \leq t_{j}<t_{i}$, instead of Eq. (13).

Figure 3 shows a schematic example of a pixel connection map in which two target echoes A and B intersect. The gray and white pixels corresond to targets A and B. To simplify the figure, only the pixel connections for target A are shown. Using a pixel connection map, the echoes from the same target

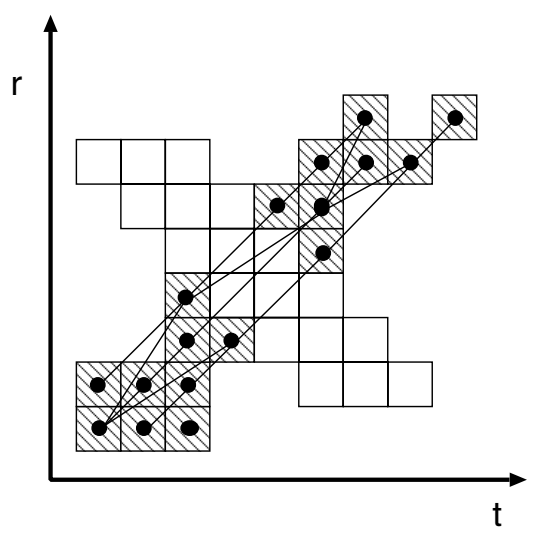

Fig. 3. An example of a pixel connection map for two targets (gray pixels for A and white pixels for B).

is associated even if the echo from the same target is separated due to shadowing by the other target.

\section{B. Complex Number Propagation Algorithm}

Next, we introduce the method that automatically separates multiple echoes using the pixel connection maps $R_{i}, R_{i}^{\prime}, L_{i}$ and $L_{i}^{\prime}$ that were calculated in step 2. The pixel connection maps are not completely accurate; pixels belonging to different targets can be erroneously connected. The algorithm proposed below benefits from statistical averaging effects to suppress such errors. This algorithm forms a new image by repetitively updating a few pixels at a time. We call this image the "connection propagation image", denoted $I_{n}$, where $n=0,1, \cdots$ is the iteration number.

First, we initialize the connection propagation image $I_{0}$. A uniformly distributed random variable $0 \leq \psi<2 \pi$ is chosen independently for each pixel to generate a unit complex number $\mathrm{e}^{j \psi}$; if the corresponding amplitude for the pixel is less than the threshold, a zero value is assigned to the pixel of the connection propagation image. Thus

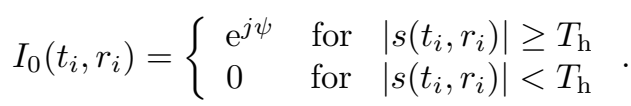

In each iteration, we randomly pick a pixel index $i \in$ $\left\{1,2, \cdots, M_{\mathrm{p}}\right\}$ from the connection propagation image, where $M_{\mathrm{p}}$ is the number of pixels in the connection propagation image. Then the pixels are updated if $t_{i} \leq(1+\alpha) T_{\max } / 2$ as

$$
\begin{aligned}
I_{n}\left(t_{i}, r_{i}\right) & =\left(I_{n-1}\left(t_{i}, r_{i}\right)+I_{n-1}\left(t_{R_{i}}, r_{R_{i}}\right)\right) / 2, \\
I_{n}\left(t_{L_{i}}, r_{L_{i}}\right) & =\left(I_{n-1}\left(t_{i}, r_{i}\right)+I_{n-1}\left(t_{L_{i}}, r_{L_{i}}\right)\right) / 2,
\end{aligned}
$$

and updated if $t_{i}>(1-\alpha) T_{\max } / 2$ as

$$
\begin{aligned}
I_{n}\left(t_{i}, r_{i}\right) & =\left(I_{n-1}\left(t_{i}, r_{i}\right)+I_{n-1}\left(t_{L_{i}}, r_{L_{i}}\right)\right) / 2, \\
I_{n}\left(t_{R_{i}}, r_{R_{i}}\right) & =\left(I_{n-1}\left(t_{i}, r_{i}\right)+I_{n-1}\left(t_{R_{i}}, r_{R_{i}}\right)\right) / 2,
\end{aligned}
$$

where $T_{\max }$ is the maximum slow-time of the image. Note that although we present the propagation process using $R_{i}$ and $L_{i}$ only, we apply the same process using $R_{i}^{\prime}$ and $L_{i}^{\prime}$ as well.

Eqs. (17) and (18) mean that the complex numbers propagate to the left if the chosen pixel is on the left half of the connection propagation image. In contrast, the complex 
numbers propagate to the right with Eqs. (19) and (20) for pixels on the right half. For $i$ that satisfy $(1-\alpha) T_{\max } / 2<$ $t_{i} \leq(1+\alpha) T_{\max } / 2$, all operations Eqs. (17)-(20) are applied, which means that complex numbers propagate in both directions.

In this way, the initialized random numbers around the center of the connection propagation image propagate to both sides along the connection established in the previous subsection. Echoes corresponding to different targets have a relatively fewer number of connections, if any. This prevents the complex numbers from being mixed up across adjacent pixels that belong to different targets.

In addition, to the procedures Eqs. (17)-(20), we also make the values propagate in the range direction. The randomly chosen $i$-th pixel is updated as

$$
I_{n}\left(t_{i}, r_{i}\right)=\left(I_{n-1}\left(t_{i}, r_{i}\right)+I_{n-1}\left(t_{j}, r_{j}\right)\right) / 2,
$$

only if $\left|\theta_{i}-\theta_{j}\right|<T_{\theta}$, where $r_{j}$ is a range next to $r_{i}$, i. e. $r_{j}=r_{i} \pm \Delta r$. Here, $\Delta r$ is the image pixel size in the range direction. The process in Eq. (21) is necessary because echoes have a certain width caused by scattering from complicated human-body shapes. Without the process in Eq. (21), each segment can be erroneously separated into several band-like structures. After $n=N_{\max }$ iterations, we obtain the final connection propagation image. We use the phase of the connection propagation image $\angle I_{N_{\max }}\left(t_{i}, r_{i}\right)$ to separate the echoes.

We proposed a complex number propagation algorithm for separating targets. Here we explain the reason why we use complex numbers instead of real numbers for this purpose. If we use real numbers $x$ in a certain range (e.g. $-1 \leq x \leq 1$ ) for this algorithm, a number of random values in each target echo are averaged. After numerous iterations, these numbers converge to the midpoint $x \rightarrow 0$, which happens for all targets. Therefore, multiple targets cannot be discriminated after many iterations. In contrast, the angle of a complex number $0 \leq \angle I<2 \pi$ does not have any points to converge to. The averaged angles for multiple targets are expected to be uniformly distributed, making the target-separation capability efficient regardless of the number of iterations.

\section{Radar Measurement Setup and Data}

We recorded scattering data from two people walking using a radar system (PulsOn 400, Time Domain Corporation, $\mathrm{AL}$ ). The frequency band is from 3.1 to $5.3 \mathrm{GHz}$, and the signal is modulated by an m-sequence. The received data are compressed with the same sequence. The bandwidth $2.2 \mathrm{GHz}$ corresponds to $6.8 \mathrm{~cm}$ of range resolution, which makes a human body echo spread over multiple range bins. Therefore, a human body is seen as a distributed target in this scenario. The transmitted power is $-14.5 \mathrm{dBm}$. The transmitting and receiving antennas are dual-polarized horn antennas (model DP240, Flann Microwave Ltd., UK) with 2 to $18 \mathrm{GHz}$ bandwidth. The antennas are separated by $50.0 \mathrm{~cm}$.

First, we measured two people each walking at almost constant velocity but in opposite directions (the upper panel of Fig. 4). Target A walks from a point $1.0 \mathrm{~m}$ away from the
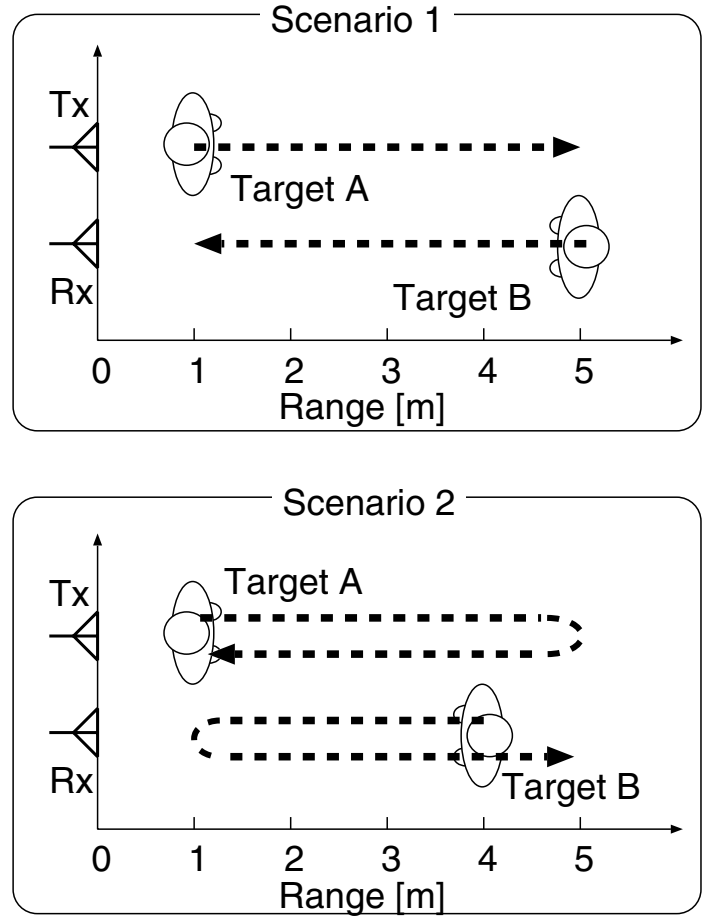

Fig. 4. Schematic of the recording setups with antennas and two people walking.

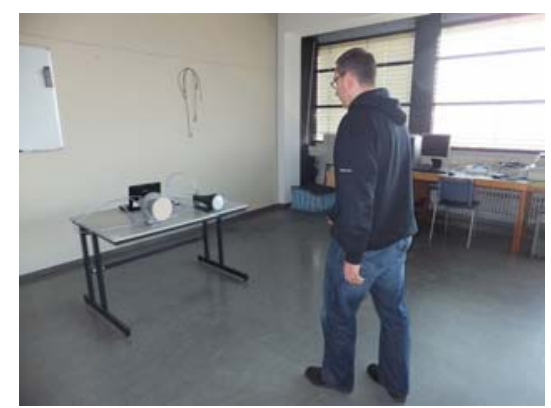

Fig. 5. Photo of the recording setup.

antennas to a point $5.0 \mathrm{~m}$ away; Target B walks in the opposite direction from $5.0 \mathrm{~m}$ away to the point $1.0 \mathrm{~m}$ away from the antennas.

Next, we measured a more complicated scenario where the two people are changing their walking velocities. The diagram of the scenario is shown in the lower part of Fig. 4. In this measurement, two people walked back and forth along the same line. Target A walks from a point $1.0 \mathrm{~m}$ away from the antennas to a point $5.0 \mathrm{~m}$ away, then back to the original point. Target B walks from a point $4.0 \mathrm{~m}$ away from the antennas to a point $1.0 \mathrm{~m}$ away, then to a point $5.0 \mathrm{~m}$ away.

The range measurement repetition frequency is $200 \mathrm{~Hz}$, and the sampling frequency is $16.39 \mathrm{GHz}$. The received signals are stored and processed afterwards. A photo of the recording setup is shown in Fig. 5.

Figure 6 shows the measured slow time-range signals $|s(t, r)|^{2}$ in the first scenario. We see two target trajectories in the image indicating forward and backward motion. They intersect at $t=4.3 \mathrm{~s}$. Although it seems obvious that there are 


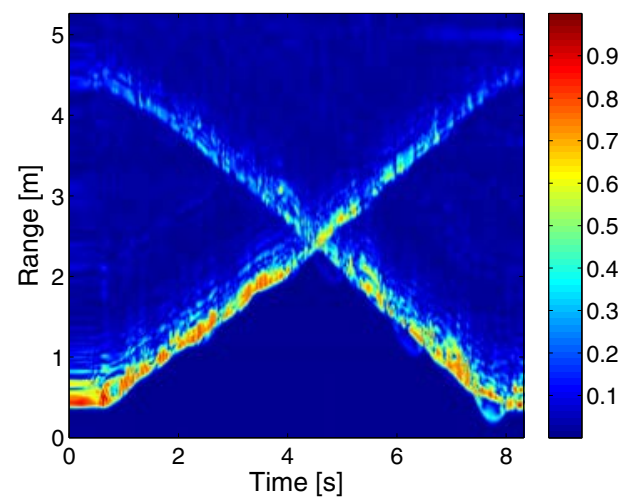

Fig. 6. Slow time-range signals $|s(t, r)|$ for two people walking in opposite directions in the first scenario.

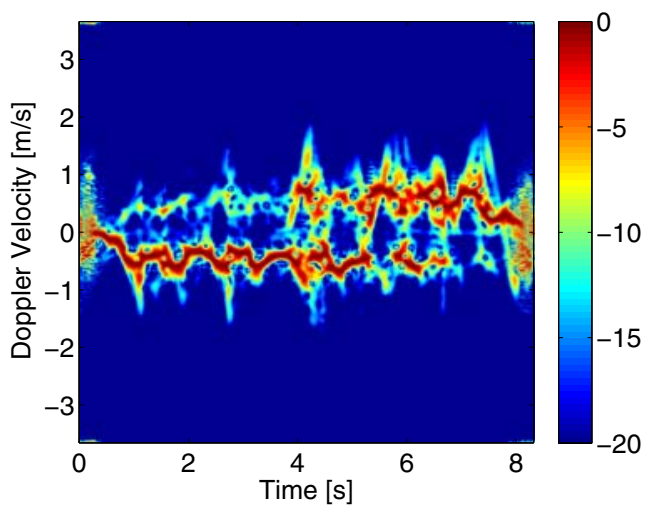

Fig. 7. SPWD $W_{\text {sum }}(t, \omega)$ of the measured signals in the first scenario (in $\mathrm{dB})$.

two targets in the image, it is not straightforward to separate these components, the principle objective described in this paper.

The SPWD image $W_{\text {sum }}(t, \omega)$, Fig. 7, of the measurement data in Fig. 6 shows that there are two trajectory components with positive and negative Doppler velocities. We set the smoothing parameters $t_{0}=0.83 \mathrm{~s}$ and $\omega_{0}=2 \pi \times 6.67 \mathrm{rad} / \mathrm{s} \mathrm{in}$ Eq. (4) for calculating spectrograms. Using opposite signs for the texture angle, echoes can be separated in the spectrogram, but this approach cannot separate the slow time-range signals, the challenge that is to be addressed.

Figure 8 shows two enlarged images of the time-range radar signals $s(t, r)$ corresponding to targets $\mathrm{A}$ and $\mathrm{B}$. Note that these images are only part of the data set, and are manually selected. The images show that two targets in the echoes have different texture directions. We use this textural feature to distinguish the two targets in the following sections.

\section{Vi. Application of the Proposed Method to MEASUREMENT DATA}

We now apply the texture angle, the pixel connection map, and the complex number propagation algorithm to the measurement data. To calculate the texture angle, $v_{0}$ is set to $1.84 \mathrm{~m} / \mathrm{s}$. A $5 \times 5$ median filter is applied to the texture angle to eliminate artifacts before calculating a pixel connection map.
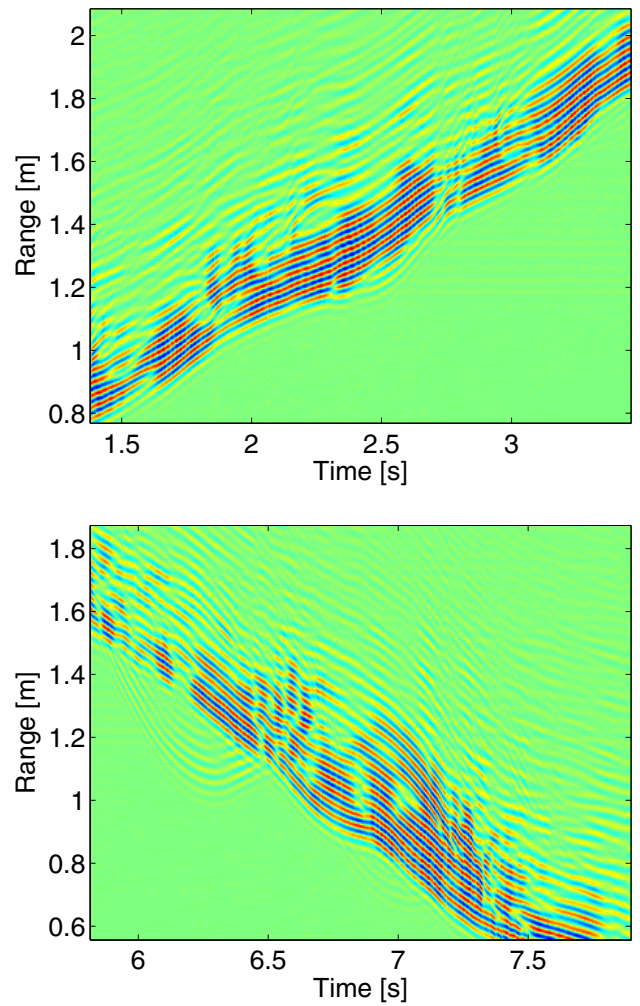

Fig. 8. Enlarged slow time-range signals $s(t, r)$ for targets A (upper image) and $\mathrm{B}$ (lower image).

For the pixel connection map, we set $T_{\mathrm{s}}=1 \mathrm{~s}$, and $\delta=0.1$ rad. For the complex number propagation algorithm, we set $T_{\mathrm{h}}=0.03 \max |s(t, r)|, \alpha=0.1$, and $T_{\theta}=\pi / 20$.

We first apply the texture angle to the measurement data in the first scenario. Figure 9 shows the texture angle of the measured signal. The texture clearly distinguishes the different targets as indicated by the red (target A) and blue (target B) tracks. The separation capability is degraded at $t<0.7 \mathrm{~s}$ and $t>7.8 \mathrm{~s}$ because neither target is moving at those times. We also see that the swinging arms of target $B$ have a positive texture angle $\theta>\pi / 2$. Compared with Fig. 7 , the texture angle has the advantage that an approximate Doppler information is given for each pixel.

As seen above, in a simple constant-velocity scenario, the texture angle alone can separate echoes in the slow timerange domain. However, in more complicated cases with targets having varying velocities, echo separation becomes more challenging.

A slow time-range radar image $|s(t, r)|$ for the second scenario is shown in Fig. 10. The echoes intersect at two points corresponding to $3 \mathrm{~s}$ and $10 \mathrm{~s}$. The SPWD of the data in Fig. 10 is displayed in Fig. 11. There, the two echoes are overlapping in the frequency domain, which makes it difficult to apply most of the conventional signal processing based on micro-Doppler information.

Next, we calculate the texture angle of the slow time-range image (Fig. 12) for the second scenario. Each of the two echoes has smooth gradation in the texture angle, which means that the target speeds change gradually. This characteristic 


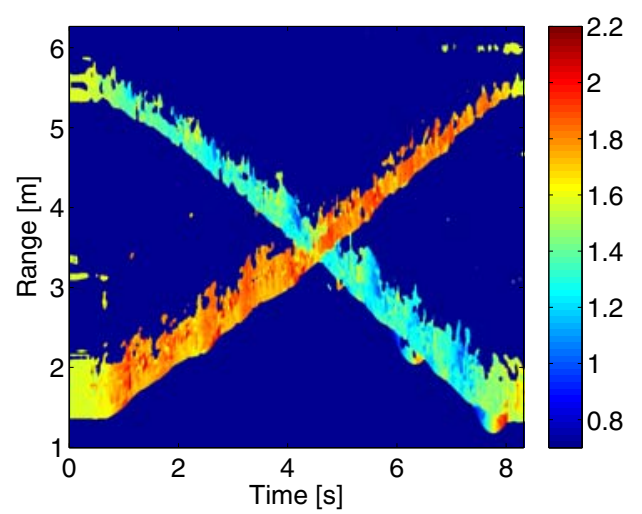

Fig. 9. Texture angle $\theta(t, r)$ for the slow time-range signals in the first scenario (in rad).

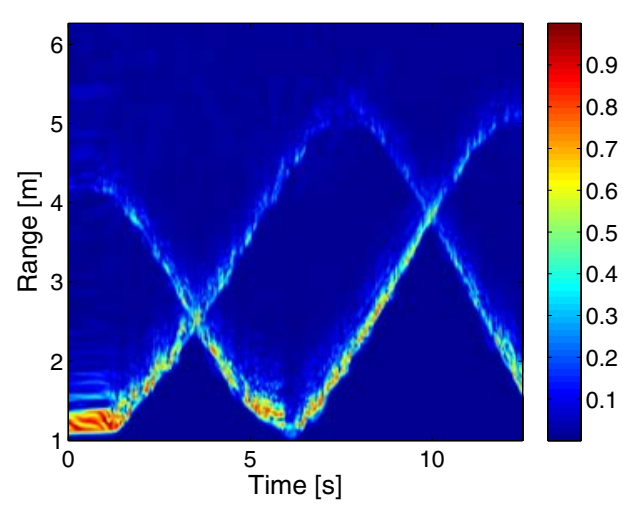

Fig. 10. Slow time-range radar image $|s(t, r)|$ measured for two people walking at time-varying speeds (the second scenario).

will be exploited by the proposed method to separate the two echoes.

The proposed pixel-connection map and complex-number propagation algorithm are applied to the texture angle image. The images in Fig. 13 show the iterative steps of the proposed method, in which the color-scaled angle of the complex value associated with each pixel is displayed. In the first image, each pixel has an independent value of the others. As the iteration

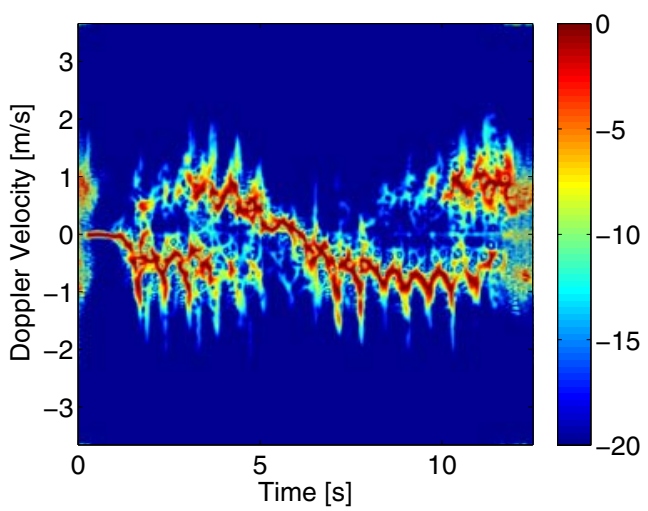

Fig. 11. SPWD $W_{\text {sum }}(t, \omega)$ of the echoes for the second scenario, two people walking with time-varying speeds (in $\mathrm{dB}$ ).

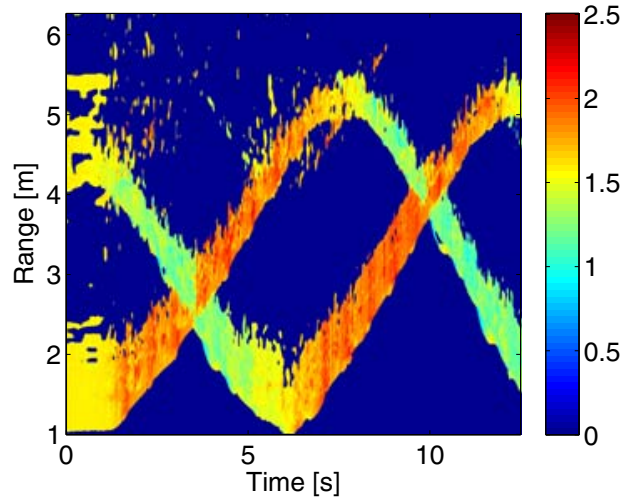

Fig. 12. Texture angle $\theta(t, r)$ calculated for the second scenario, two people walking with time-varying speeds.
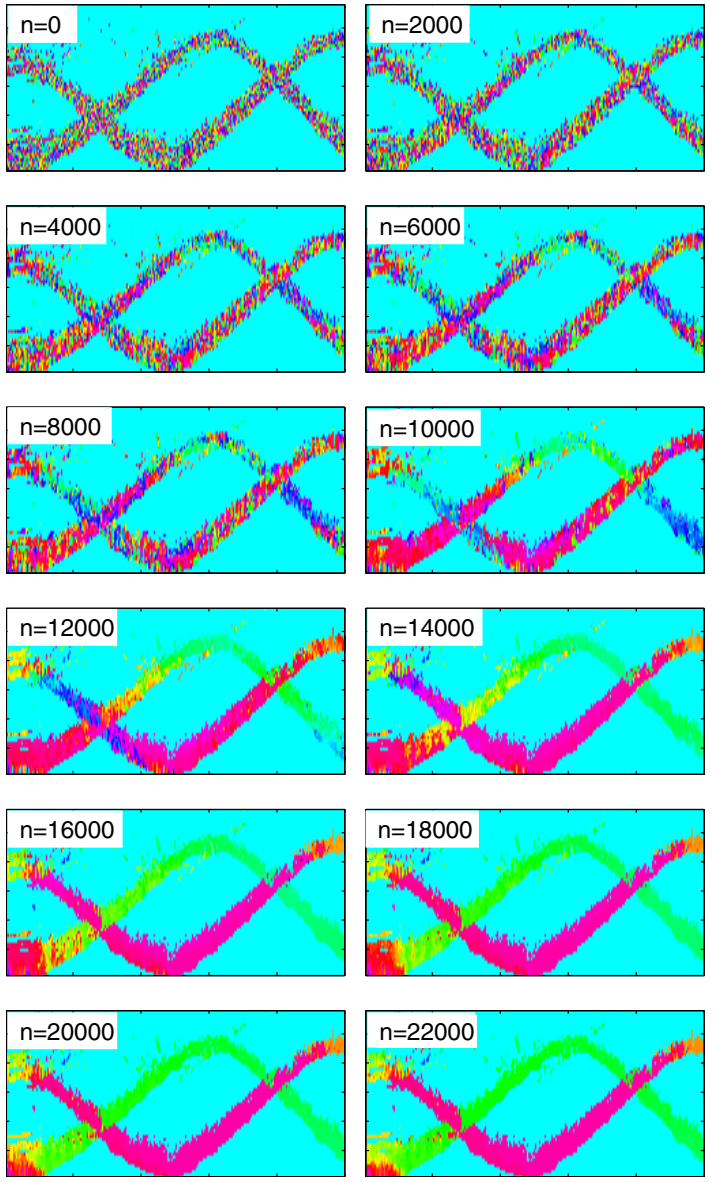

Fig. 13. Iterations in segregating the radar image using the proposed method.

progresses, the dominant colors in the middle of the image propagate outward to both sides along the echo trajectories. Even at the intersection points, pixels closely located to each other are not necessarily connected in this algorithm. This is why the color-scaled angles gravitate only to the correctly associated pixels in the image. Finally, most of the pixels in the images are correctly segregated into two dominant colors as seen in the final connection propagation image.

The final connection propagation image after $N_{\max }=$ 30000 iterations (Fig. 14) indicates that the two targets are 


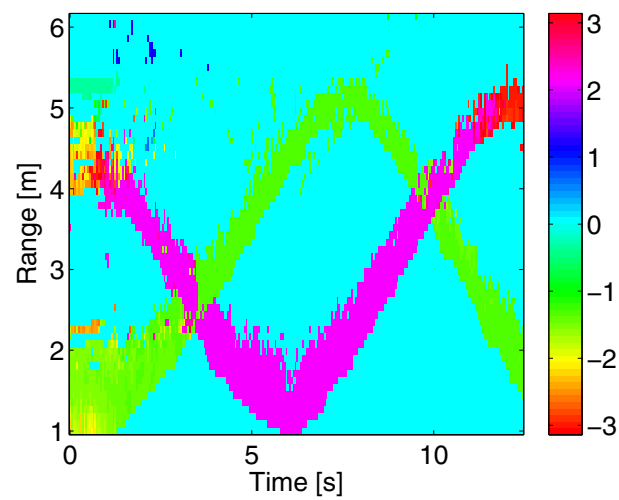

Fig. 14. Connection propagation image after applying the proposed method after 30000 iterations (in rad).

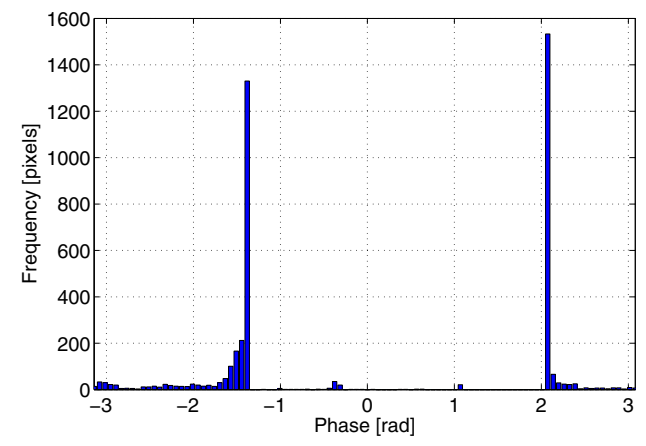

Fig. 15. Histogram of the connection propagation image in Fig.14.

clearly separated by our algorithm. A histogram of this image can be used to determine the threshold to separate the two targets. In the histogram of the image (Fig. 15), we see two significant peaks that correspond to the two targets. In this way, we do not have to know the number of targets in advance to use the proposed method. Multiple echoes are autonomously separated into different phase angles in this image.

In this case, we detected the two peaks and their corresponding values for $\angle I_{N_{\max }}\left(t_{i}, r_{i}\right)$ as $\psi_{1}=2.07 \mathrm{rad}$ and $\psi_{2}=-1.38 \mathrm{rad}$. Next, the phase of the whole connection propagation image is rotated by the average value $\psi_{\text {ave }}=$ $\left(\psi_{1}+\psi_{2}\right) / 2=0.35 \mathrm{rad}$ as $I_{\text {final }}\left(t_{i}, r_{i}\right)=I_{N_{\max }}\left(t_{i}, r_{i}\right) \mathrm{e}^{-\mathrm{j} \psi_{\text {ave }}}$ to shift the optimal threshold to 0 . Then, the slow time-range image is separated as

$$
\begin{aligned}
& s_{1}(t, r)=\left\{\begin{array}{ccc}
s(t, r) & \text { if } & \angle I_{\text {final }}\left(t_{i}, r_{i}\right)>0 \\
& 0 & \text { otherwise }
\end{array},\right. \\
& s_{2}(t, r)=\left\{\begin{array}{ccc}
s(t, r) & \text { if } & \angle I_{\text {final }}\left(t_{i}, r_{i}\right) \leq 0 \\
& 0 & \text { otherwise }
\end{array} .\right.
\end{aligned}
$$

With the proposed algorithm, the signals in the image of Fig. 10 are for the most part clearly separated, as shown in Fig. 16 although some undesired components are seen in the lower image. Further, some data are missing around $3.5 \mathrm{~s}$ and $10 \mathrm{~s}$ that correspond to the intersection points.

The spectrograms of the two separated signals are shown in Fig. 17. The detailed micro-Doppler information of the two targets is now retrieved by separating the echoes. The irregular
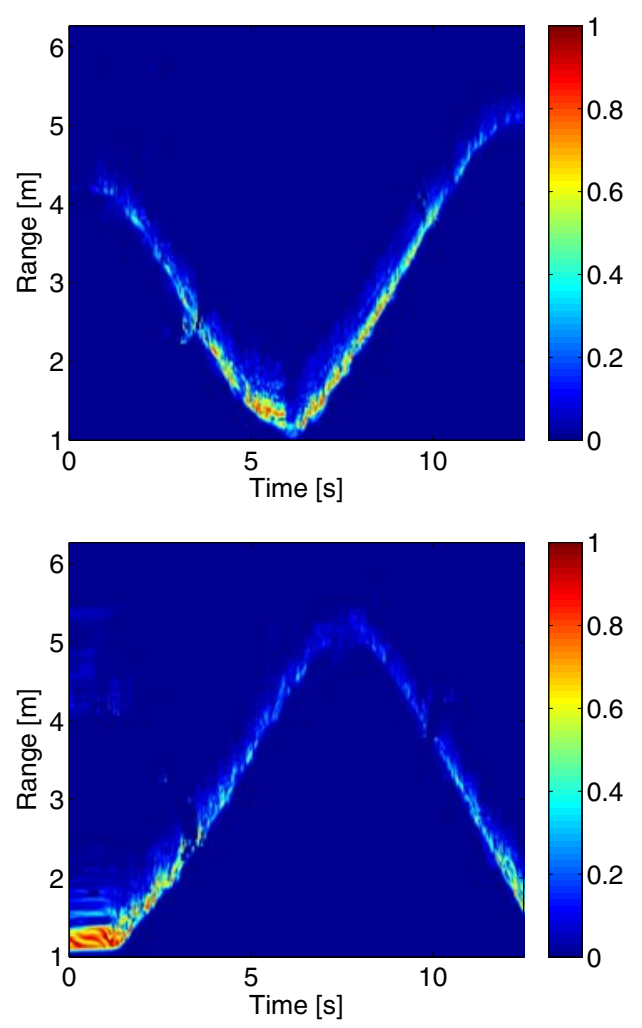

Fig. 16. Separated echoes $s_{1}(t, r)$ and $s_{2}(t, r)$ using the proposed complex number propagation algorithm.

spots seen at $3.5 \mathrm{~s}$ and $10 \mathrm{~s}$ are caused by the missing data, also seen in Fig. 16. These images show outstanding advantage compared with the original SPWD shown in Fig. 11.

\section{Performance in Complicated Scenarios}

In this section, we present the analysis of the performance of the proposed algorithm to various situations of greater difficulty. First, we investigate the algorithm's capability to separate two targets of similar line-of-sight speeds. With such concurrences, the pixel connection map can erroneously connect the two different echoes. To investigate this problem, we generate simulated radar images using measurement data for a single target as $s(t, r)+s\left(t,(1+a)\left(r-r_{0}\right)+r_{0}\right)$, where $s(t, r)$ contains only a single target moving approximately at $0.7 \mathrm{~m} / \mathrm{s}$. The parameter $a$ is the difference of targets' speeds normalized to the original speed $0.7 \mathrm{~m} / \mathrm{s}$. The proposed algorithm is applied to the simulated radar images with various initial values for the complex number propagation procedure. Some of the synthesized radar images are shown in Fig. 18.

We calculate the success rate of target separation using Monte Carlo simulations. Because the proposed algorithm can underestimate the number of targets depending on the initial values of the complex propagation algorithm, the success rate is determined as the maximum number of targets separated when using the proposed algorithm $N_{\mathrm{a}}$ times. Fig. 19 shows the success rate of target separation for $N_{\mathrm{a}}=3$ calculated from 100 realizations. This figure indicates that $a>0.94$ i.e. the difference of target speeds must be larger than 0.66 $\mathrm{m} / \mathrm{s}$ to achieve a success rate of 0.9 . 

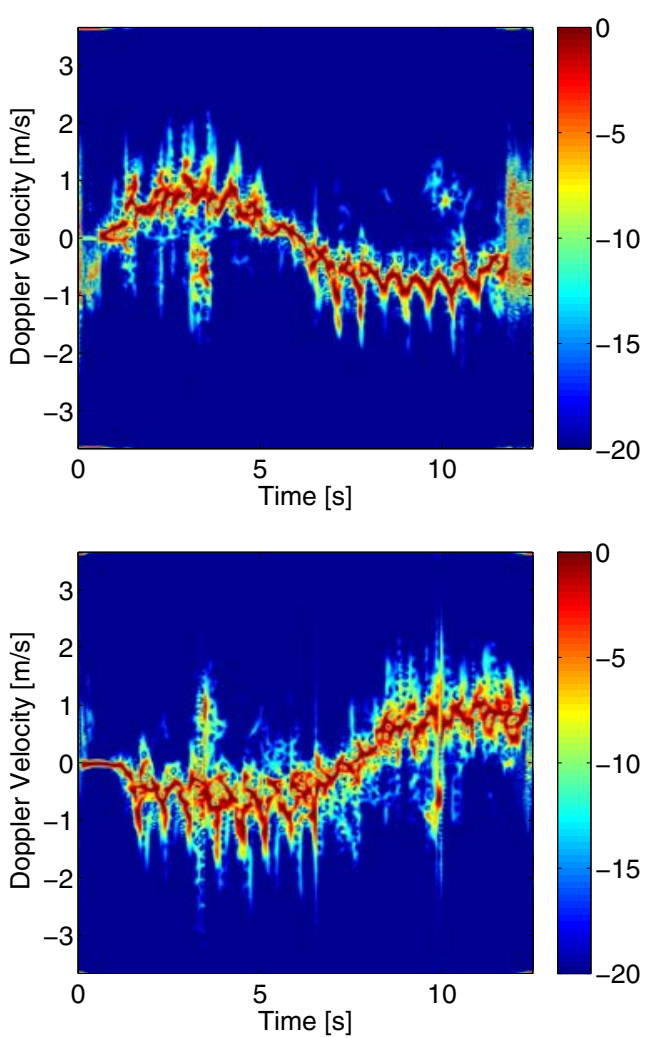

Fig. 17. SPWDs $W_{\text {sum }}(t, \omega)$ of separated echos using the proposed complex number propagation algorithm (in $\mathrm{dB}$ ).
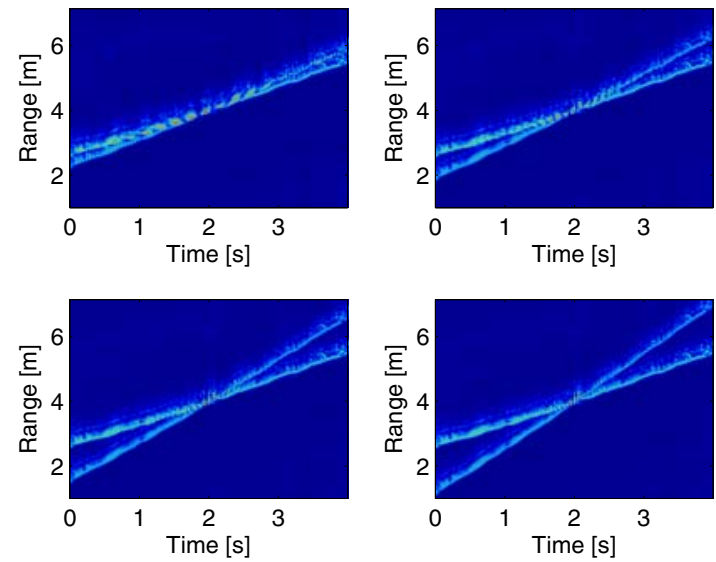

Fig. 18. Synthesized radar images for $a=0.25,0.5,0.75$ and 1.0 (upper left, upper right, lower left and lower right).

We then apply the proposed algorithm to a scenario with three targets. Fig. 20 shows the radar image for three people walking at almost the same speeds. Fig. 21 shows the separated target echoes. In this way, the algorithm can handle more than three targets, only if the speeds of targets are not close to each other at intersection points. The focus of future work is to develop a method to find optimal threshold values to separate more than two targets.

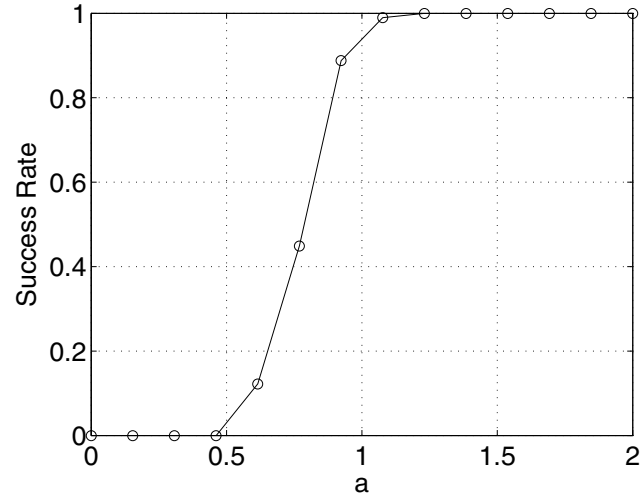

Fig. 19. Success rate of target separation for various $a$.

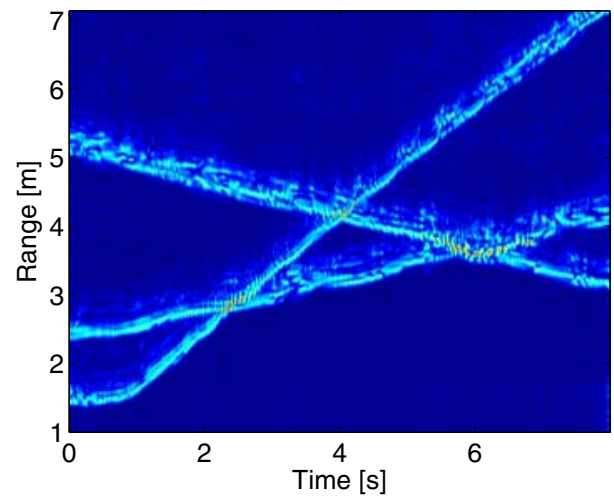

Fig. 20. Slow time-range signals $|s(t, r)|$ for three targets.

Finally, we mention some parameter settings to improve the tolerance against close speeds. The parameter $T_{\mathrm{s}}$ in Eq. (13) should be larger, because target echoes overlap for a long time when targets move at similar speeds. The parameter $\delta$ in Eq. (14) must have a smaller value so that the pixel connection map does not erroneously connect pixels that belong to different targets but have close texture angles.

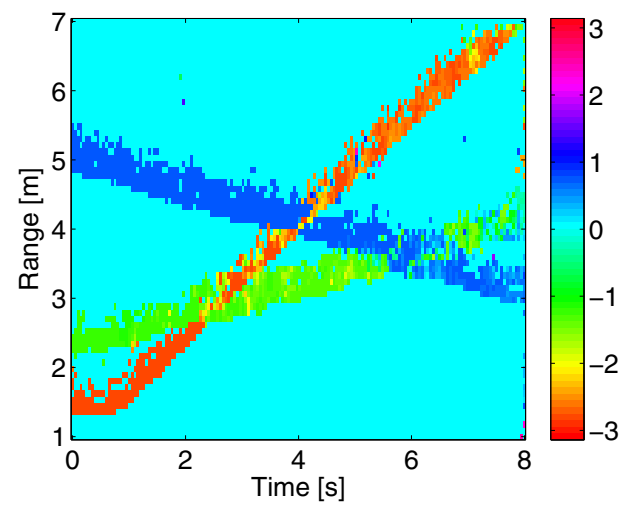

Fig. 21. Connection propagation image for three targets (in rad). 


\section{CONCLUSION}

We proposed a new algorithm for separating multiple distributed targets using UWB radar. The proposed method calculates the texture angle to estimate an approximate lineof-sight speed of the target at each point of the signals. Targets with different speeds have different textures in the slow time-range image. The texture angle was applied to the measurement of two targets walking at almost constant speed, and was demonstrated to be effective in labeling multiple echoes. We further developed an extended algorithm based on the texture angle for separating targets moving at varying speeds. In the algorithm, we calculate a pixel-connection map that represents pixels connected by having similar texture angles. Moreover, a pair of pixels is chosen such that their relative position does not contradict the value of the texture angle corresponding to the Doppler velocity of each pixel. Finally, randomly distributed complex values are numerically propagated to adjacent connected pixels. This algorithm does not require a prior knowledge of the number of targets. The randomly assigned complex numbers automatically propagate and merge into multiple segments. We have demonstrated that the proposed algorithm can successfully separate two motionvarying targets from echoes. We also obtained a minimum condition for speed differences that would enable target signals to be successfully separated. As long as this condition is satisfied, the algorithm can separate as many as three targets.

\section{ACKNOWLEDGEMENT}

This study was supported in part by the Japan Society for the Promotion of Science (JSPS) Postdoctoral Fellowships for Research Abroad (High-resolution imaging for human bodies with UWB radar using multipath echoes).

\section{REFERENCES}

[1] Y. Kim and H. Ling, "Human activity classification based on microDoppler signatures using a support vector machine," IEEE Transactions on Geoscience and Remote Sensing, vol. 47, no. 5, pp. 1328-1337, May 2009.

[2] A. Sona, R. Ricci and G. Giorgi, "A measurement approach based on micro-Doppler maps for human motion analysis and detection," Proc. IEEE International Instrumentation and Measurement Technology Conference, pp. 354-359, May 2012.

[3] D. Tahmoush and J. Silvious, "Simplified model of dismount microDoppler and RCS," Proc. IEEE Radar Conference, pp. 31-34, May 2010.

[4] P. Molchanov, J. Astola and A. Totsky, "Frequency and phase coupling phenomenon in micro-Doppler radar signature of walking human," Proc. 19th International Radar Symposium, pp. 49-53, May 2012.

[5] J. Li, Z. Zeng, J. Sun and F. Liu, "Through-wall detection of human being's movement by UWB radar," IEEE Geoscience and Remote Sensing Letters, vol. 9, no. 6, pp. 1079-1083, Nov. 2012.

[6] C.-P. Lai, R. M. Narayanan, Q. Ruan and A. Davydov, "Hilbert-Huan transform analysis of human activities using through-wall noise and noiselike radar,' IET Radar Sonar Navig., vol. 2, no. 4, pp. 244-255, 2008.

[7] A. G. Yarovoy, L. P. Ligthart, J. Matuzas and B. Levitas, "UWB radar for human being detection," IEEE A\&E Systems Magazine, pp. 36-40, May 2008.

[8] K. Saho, T. Sakamoto, T. Sato, K. Inoue and T. Fukuda, "Experimental study of real-time human imaging using UWB Doppler radar interferometry," Prof. 6th European Conference on Antennas and Propagation, pp. 3495-3497, 2011.

[9] K. Saho, T. Sakamoto, T. Sato, K. Inoue and T. Fukuda, "Pedestrian classification based on radial velocity features of UWB Doppler radar images" Proc. 2012 International Symposium on Antennas and Propagation, pp. 90-93, 2012.
[10] Y. Wang and A. E. Fathy, "Micro-Doppler signatures for intelligent human gait recognition using a UWB impulse radar," Proc. pp. 21032106, 2011.

[11] S.-H. Chang, R. Sharan, M. Wolf, N. Mitsumoto, and J. W. Burdick, "An MHT algorithm for UWB radar-based multiple human target tracking," Proc. IEEE International Conference on Ultra-Wideband, pp. 459-463, Sep. 2009.

[12] Y. He, F. le Chevalier, and A. G. Yarovoy, "Association of rangedoppler video sequences in multistatic UWB radar for human tracking," Proc. 2012 9th European Radar Conference (EuRAD), pp. 218-221, 2012.

[13] A. Hoogs, R. Collins, R. Kaucic, and J. Mundy, "A common set of perceptual observables for grouping, figure-ground discrimination, and texture classification," IEEE Transactions on Pattern Analysis and Machine Intelligence, Vol. 25, No. 4, pp. 458-474, 2003.

[14] A. F. Said and L. J. Karam, "Multi-Region Texture Image Segmentation Based on Constrained Level-Set Evolution Functions," Proc. IEEE 13th Digital Signal Processing Workshop and 5th IEEE Signal Processing Education Workshop, pp. 664-668, 2009.

[15] Y. Deng and B. S. Manjunath, "Unsupervised segmentation of colortexture regions in images and video," IEEE Transactions on Pattern Analysis and Machine Intelligence, Vol. 23, No. 8, pp. 800-810, 2001.

[16] I. Karoui, R. Fablet, J.-M. Boucher, J. Augustin, "Variational RegionBased Segmentation Using Multiple Texture Statistics," IEEE Transactions on Image Processing, Vol. 19, No. 12, pp. 3146-3156, 2010. 